\title{
Estudo experimental da inibição da proliferação vitreorretiniana pelo uso da hiperecina
}

\author{
Experimental study of vitreoretinal proliferation inhibition \\ by the use of hypericin
}

\author{
Roberto Auguusto Fernandes Machado ${ }^{1}$ \\ Antonio Marcelo Barbante Casella ${ }^{2}$ \\ Marcus Rudolph Malaguido ${ }^{3}$ \\ Ana Paula Miyagusko Taba Oguido ${ }^{4}$
}

\begin{tabular}{|l|}
\hline RESUMO \\
\hline Objetivos: Induzir a produção de membranas vitreorretinianas em mode- \\
lo de trauma ocular animal. Avaliar a inibição do desenvolvimento da \\
proliferação vitreorretiniana (PVR) com o uso de hiperecina. Métodos: \\
Estudo Experimental. Foram utilizados 19 coelhos machos pigmentados \\
adultos com peso entre 2.000 e 3.000 gramas. Todos submetidos a \\
modelo de trauma com dispase associada à diatermia da retina para \\
indução de membranas de PVR. Separados randomicamente para rece- \\
berem hiperecina (10 $\mu$ M em 0,1 ml) ou solução salina (0,1 ml) como \\
placebo. Avaliados clinicamente no sétimo, décimo quarto, vigésimo pri- \\
meiro e vigésimo oitavo dias de pós-operatório com oftalmoscopia in- \\
direta e retinografia colorida digitalizada. O grau de PVR foi classificado \\
em estágios (de 0 a 7) segundo Hida e colaboradores. Resultados: A \\
formação de membranas esteve presente em 79\% dos olhos, sendo $100 \%$ \\
nos olhos do grupo placebo e $60 \%$ nos olhos do grupo tratamento (hipe- \\
recina). A comparação entre as médias dos estágios de PVR entre os grupos \\
mostrou diferença estatisticamente significativa, com valor p=0,0321 \\
pelo teste Wilcoxon. Conclusões: Omodelo de trauma com uso de dispase \\
e diatermia da retina produz membranas vitreorretinianas. A hiperecina \\
mostrou-se eficaz na diminuição do aparecimento e progressão do PVR.
\end{tabular}

Descritores: Vitreorretinopatia proliferativa/etiologia; Vitreorretinopatia proliferativa/prevenção \& controle; Antidepressivos/uso terapêutico; Descolamento de retina; Traumatismos oculares; Cicatrização de feridas; Modelos animais de doenças; Coelhos
Trabalho realizado no Departamento de Clínica Cirúrgica da Universidade Estadual de Londrina - UEL Londrina (PR) - Brasil.

${ }^{1}$ Médico Oftalmologista, Mestre em Medicina e Ciências da Saúde pela Universidade Estadual de Londrina - UEL Londrina (PR) - Brasil.

${ }^{2}$ Doutor, Professor Adjunto de Oftalmologia da UEL Londrina (PR) - Brasil.

Médico Oftalmologista, Mestre em Medicina e Ciências da Saúde pela UEL - Londrina (PR) - Brasil.

${ }^{4}$ Médica Oftalmologista, Mestre em Medicina e Ciências da Saúde pela UEL - Londrina (PR) - Brasil.

Endereço para correspondência: Marcus Rudolph Malaguido. Rua Dr. Desembargador Clotário Portugal, 749, Apto 502-C - Apucarana (PR)

CEP 86800-020

E-mail: marcus.malaguido@uol.com.br

Recebido para publicação em 23.05.2008

Versão recebida em 07.03.2009

Aprovação em 04.08.2009

Trabalho apresentado ao Programa de Pós-Graduação em Medicina e Ciências da Saúde da Universidade Estadual de Londrina no ano de 2002, como requisito parcial para obtenção do título de mestre do primeiro autor.

\section{INTRODUÇÃO}

A proliferação vitreorretiniana (PVR) é a formação de membranas celulares em ambas as faces da retina descolada com propriedades contráteis, levando a uma tração da retina e um descolamento de retina recidivante ${ }^{(1-2)}$. É a maior causa de insucesso terapêutico nos casos de descolamento de retina $^{(1)}$. Tem incidência entre 5 a $10 \%$ dos descolamentos de retina, com média de $7 \%{ }^{(3)}$. A presença de PVR piora os prognósticos anatômico e funcional no pós-operatório dos descolamentos de retina ${ }^{(4-10)}$, com resultados visuais não refletindo os resultados anatômicos ${ }^{(8,10)}$.

Modelos animais de PVR têm sido produzidos para estudo de fatores e células envolvidas na sua gênese e drogas para interferir em suas diversas fases ${ }^{(11-16)}$. Vários grupos de drogas têm sido utilizados, dentre os quais se destacam os corticosteróides ${ }^{(14,17-19)}$, anti-inflamatórios não esteróides ${ }^{(20)} \mathrm{e}$ drogas antiproliferativas como o 5-fluoruracil ${ }^{(11-14,16,18-20)}$.

Hida et al. ${ }^{(21)}$ desenvolveram um modelo de trauma ocular utilizando compressão gasosa do vítreo com perfluorpropano associada à injeção de 
25.000 fibroblastos homólogos. No mesmo estudo, relatam sua classificação de PVR experimental em coelhos, dividindo em oito estágios (0 a 7).

Tahara et al. ${ }^{(22)}$ publicaram um estudo utilizando a hiperecina para inibição da PVR, mostrando resultados satisfatórios, mas em modelo de trauma que usa células estranhas ao olho (injeção de fibroblastos).

Também utilizando hiperecina, Gao et al. reduziram a formação de PVR em um modelo de trauma em coelhos que usa injeção intravítrea de plasma com plaquetas ${ }^{(23)}$.

A hiperecina é um diono policíclico aromático derivado de plantas do gênero Hypericum com atividade em vários campos da medicina natural na Europa, e também aplicações na medicina tradicional como tratamento de tumores, depressão, cicatrizante, antiviral, entre outros. O hipérico é um gênero de plantas nativas da Europa, África e Ásia que hoje podem ser encontradas em outras partes do mundo ${ }^{(24)}$. Um estudo descreveu a inibição irreversível do receptor de fator de crescimento epidérmico (EGF-R), serina/tirosinaquinase, proteinoquinase $\mathrm{C}$ e proteinoquinase CK2 com o uso de hiperecina. Cita ainda que seu mecanismo de inibição não está totalmente esclarecido, mas seria através de mecanismo fotossensibilizante tipo oxigênio-independente ${ }^{(25)}$.

O nosso estudo pretende induzir a produção de membranas vitreorretinianas em modelo de trauma ocular animal que não usa adição de células estranhas ao olho, e avaliar a inibição do desenvolvimento da proliferação vitreorretiniana com o uso de hiperecina.

\section{MÉTODOS}

\section{Tipo de estudo: estudo experimental}

Foram utilizados 19 coelhos machos pigmentados, adultos, pesando entre 2 e $3 \mathrm{~kg}$, que foram mantidos em biotério da Universidade Estadual de Londrina, onde receberam ração comercial e água à vontade e foram tratados conforme as normas brasileiras para uso de animais em atividades científicas (segundo a Lei $\mathrm{n}^{\circ}$ 11.794/08 e o Conselho Nacional de Controle de Experimentação Animal - CONCEA). Para a realização do experimento foi utilizado apenas o olho direito de cada animal.

Os animais receberam anestesia geral intramuscular com xilazina $7 \mathrm{mg} / \mathrm{kg}$ (Virbaxyl $^{\circledR}$ - Virbac - BR) e ketamina $35 \mathrm{mg} / \mathrm{kg}$ (Francotar ${ }^{\circledR}$ - Virbac - BR), associada ao uso tópico de proparacaína (Anestalcon ${ }^{\circledR}$ - Alcon - BR). A dilatação pupilar foi conseguida com a instilação no fundo de saco conjuntival de fenilefrina a 10\% (Fenilefrina ${ }^{\circledR}$ - Allergan - BR) associado à tropicamida 2\% (Mydriacyl ${ }^{\circledR}$ - Allergan - BR).

Com o auxílio de um microscópio cirúrgico, foi realizada uma esclerotomia a $2 \mathrm{~mm}$ do limbo com esclerótomo $20 \mathrm{G}$ (Alcon Laboratories - USA). Utilizando uma lente de contato sobre a córnea para visualização da retina e raias medulares, foi introduzido, através da esclerotomia, uma ponta de endo- diatermia Mentor $^{\circledast}$ (Mentor Inc - USA) e realizadas 12 marcas inferiores à raia medular agrupadas de quatro em quatro. Estas marcas foram realizadas com potência de $12 \%$ do equipamento de endodiatermia e foram consideradas válidas quando houvesse uma mudança da coloração da retina para uma cor esbranquiçada. Em seguida, a esclerotomia era suturada com fio de poligalactina 7-0 (Vicryl $^{\circledR}$ - BR) com pontos separados (dois pontos). Após a sutura, uma agulha $13 \mathrm{X} 4,5$ era introduzida no mesmo local da esclerotomia e $0,1 \mathrm{ml}$ de solução contendo dispase a 0,05 UI (Dispase ${ }^{\circledast}$ - Gibco - USA) era injetada na posição anterior ao nervo óptico.

Todos os animais foram submetidos ao procedimento descrito acima. Logo em seguida, outra agulha 13 X 4,5 era introduzida na esclerotomia e $0,1 \mathrm{ml}$ de outra substância era injetada na região anterior ao nervo óptico. Esta solução estava contida em seringa numerada e a substância que cada seringa continha estava anotada em folha de papel que ficou lacrada dentro de um envelope que só foi aberto no término do acompanhamento dos grupos.

Os grupos foram aleatoriamente divididos para receber a injeção, sendo 10 olhos recebendo a solução de hiperecina (Hypericin ${ }^{\circledR}$ Sigma-Aldrich - USA) na dose de $10 \mu \mathrm{mol}$, conforme Tahara et al. ${ }^{(22)}$ em $0,1 \mathrm{ml}$. Outros nove olhos receberam solução salina pura em $0,1 \mathrm{ml}$.

A hiperecina foi diluída em soro com o auxílio de sonicação para melhor homogeneização da solução. Foi esterelizada por passagem em filtros milipore em capela especial.

Após os procedimentos foi usada pomada de antibiótico ciprofloxacina (Ciloxan ${ }^{\circledR}$ - Alcon) no olho operado, e o animal foi para recuperação anestésica no biotério. Nenhum outro medicamento foi utilizado até o término do estudo, com exceção dos colírios para dilatação pupilar.

No acompanhamento clínico foi realizada oftalmoscopia binocular indireta com dilatação pupilar através do uso de colírios de fenilefrina 10\% (Fenilefrina ${ }^{\circledR}$ - Allergan - BR) e tropicamida (Mydriacyl ${ }^{\circledR}$ - Alcon - BR) no $7^{\circ}, 14^{\circ}, 21^{\circ}$ e $28^{\circ}$ dias de pós-operatório. O grau de descolamento de retina e PVR foram classificados segundo a classificação de Hida et al. ${ }^{(21)}$ (Tabela 1). Também foi realizada retinografia colorida com o retinógrafo Topcon TRC $50 \mathrm{com}$ sistema de digitalização de imagens da Ophthalmics Image System (OIS inc - USA).

$\mathrm{Na}$ análise estatística foi utilizado o programa SAS e o teste de Wilcoxon para comparação entre duas amostras independentes e dados não paramétricos. O nível de significância adotado foi de $5 \%(\mathrm{P}<0,05)$.

\section{RESULTADOS}

O exame de fundoscopia mostrou desde ausência de desenvolvimento de membranas e tração sobre a retina até descolamento total da retina com fibrose da mesma, ou seja, todos os estágios, segundo Hida et al. ${ }^{(21)}$, foram observados (Figura 1).

Durante as quatro semanas de acompanhamento foi observada a indução de PVR em 79\% dos olhos de todos os coelhos 


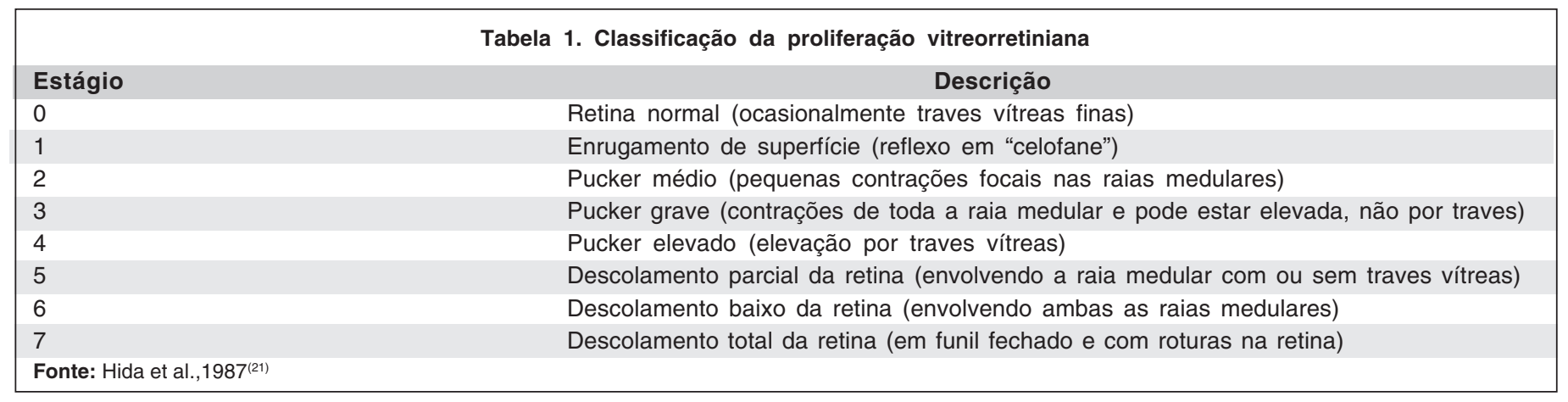
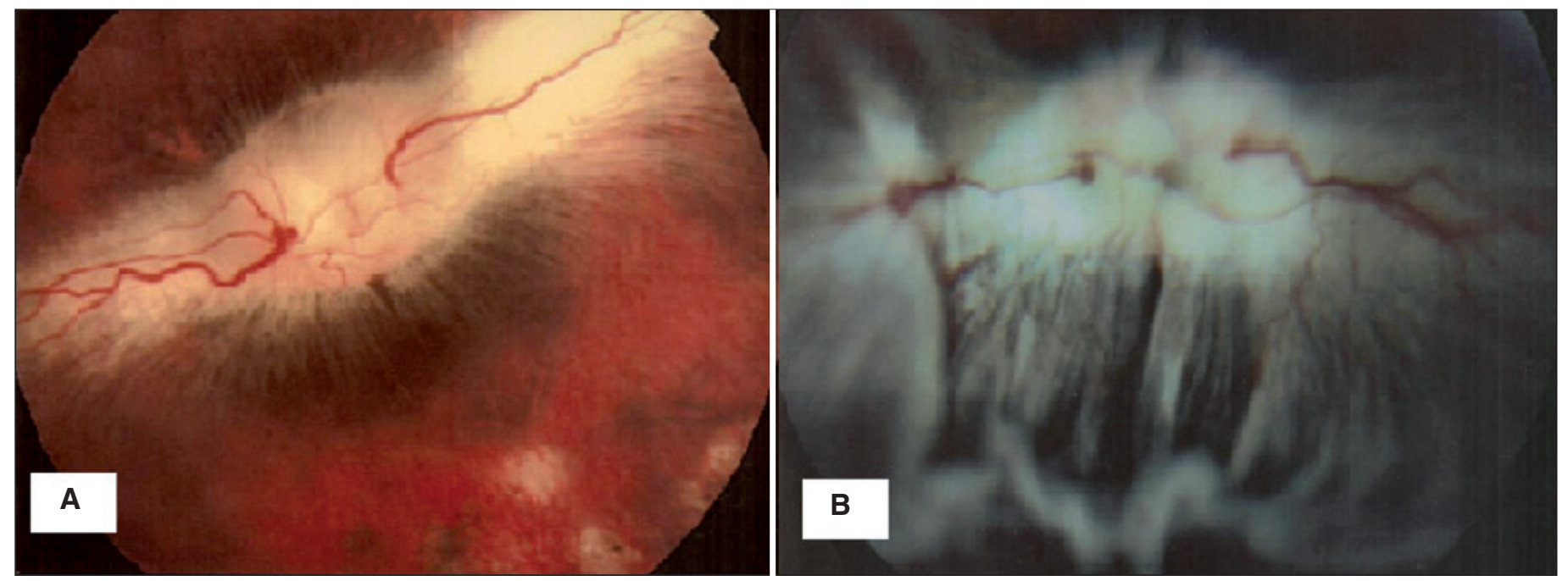

Figura 1 - A) Coelho no 26 no 21ำ dia de pós-operatório (estágio 1); B) Coelho no 23 no 7ํ dia de pós-operatório (estágio 6)

(os dois grupos juntos), sendo a sua incidência igual a $100 \%$ no grupo placebo e $60 \%$ no grupo tratamento.

Os resultados da classificação de PVR (Hida et al., 1987)(21) em cada animal nos quatro dias de exame de pós-operatório estão resumidos na tabela 2 .

A média aritmética do grau de PVR pela classificação de Hida foi igual a 3,56 no grupo placebo (seringa com solução salina) e 2,6 no grupo tratamento (hiperecina), pelos dados obtidos no vigésimo oitavo dia de pós-operatório (Figura 2).

Houve diferença estatisticamente significativa entre os dois grupos quando compararmos as posições da classificação de Hida et al. ${ }^{(21)}$ no $28^{\circ}$ dia de pós-operatório.

A progressão do PVR foi estatisticamente maior no grupo placebo quando comparado com o grupo tratamento. $\mathrm{O}$ valor $\mathrm{P}$ encontrado $(0,0321)$ foi considerado estatisticamente significante.

\section{DISCUSSÃO}

Frenzel et al. ${ }^{(26)}$ descreveram um modelo experimental em coelhos, utilizando dispase associada ou não à diatermia ou roturas na retina. Acreditam que a dispase possa favorecer o acesso de células do EPR ao vítreo. Este modelo apresenta a vantagem de desenvolver PVR somente com células nativas do olho e não células injetadas que levariam à ativação de outras vias da gênese do PVR, além de outras vantagens como menor trauma ocular, permanência da transparência do cristalino e da câmara anterior e boa dilatação pupilar ${ }^{(26)}$.

A dispase é uma protease neutra, uma metaloenzima produzida pelo Bacillus polymyxa. No olho, atua em várias vias do PVR. Entre elas, destacam-se: a quebra da barreira hematorretiniana permitindo a liberação de fatores de crescimento, citocinas e células inflamatórias; como metaloproteinase, degradando a matriz extracelular, permitindo o movimento das células durante o processo de remodelação, migração e metástases, além de degradar a matriz extracelular em fragmentos que são quimiotáticos; ativação do sistema plasminogênioplasmina e do hialuronan, um componente do vítreo que pode estar associado diretamente com a migração de células como o ligand CD44, que é expresso por fibroblastos, células de Müller e células do EPR cultivadas; e ainda outras vias que levam à liberação de fator de crescimento derivado das plaquetas ${ }^{(26)}$.

$O$ resultado deste estudo pode ter relação com o fato de que a inibição da proteinoquinase $\mathrm{C}$ leva a uma regulação para baixo no processo de proliferação vitreorretiniana. 


\begin{tabular}{|c|c|c|c|c|c|}
\hline \multicolumn{6}{|c|}{ ITabela 2. Distribuição dos animais e resultados } \\
\hline \multirow[t]{2}{*}{ Animal número } & \multirow[t]{2}{*}{ Ampola } & \multicolumn{4}{|c|}{ Estágio de PVR* ${ }^{\star}$} \\
\hline & & $7^{\circ}$ dia & $14^{\circ} \mathrm{dia}$ & $21^{\circ}$ dia & $28^{\circ} \mathrm{dia}$ \\
\hline 3 & Solução salina & 1 & 2 & 2 & 3 \\
\hline 14 & Solução salina & 1 & 2 & 2 & 1 \\
\hline 18 & Solução salina & 2 & 2 & 2 & 3 \\
\hline 21 & Solução salina & 1 & 1 & 1 & 1 \\
\hline 22 & Solução salina & 2 & 2 & 2 & 3 \\
\hline 23 & Solução salina & 6 & 7 & 7 & 7 \\
\hline 27 & Solução salina & 5 & 5 & 5 & 5 \\
\hline 28 & Solução salina & 7 & 7 & 7 & 7 \\
\hline 32 & Solução salina & 2 & 1 & 2 & 2 \\
\hline 15 & Hiperecina & 1 & 2 & 2 & 2 \\
\hline 16 & Hiperecina & 1 & 0 & 0 & 0 \\
\hline 17 & Hiperecina & 5 & 5 & 5 & 6 \\
\hline 19 & Hiperecina & 1 & 2 & 2 & 1 \\
\hline 20 & Hiperecina & 0 & 0 & 0 & 0 \\
\hline 24 & Hiperecina & 0 & 0 & 0 & 0 \\
\hline 25 & Hiperecina & 5 & 6 & 6 & 6 \\
\hline 26 & Hiperecina & 1 & 1 & 1 & 0 \\
\hline 30 & Hiperecina & 5 & 6 & 7 & 7 \\
\hline 31 & Hiperecina & 2 & 4 & 4 & 4 \\
\hline
\end{tabular}

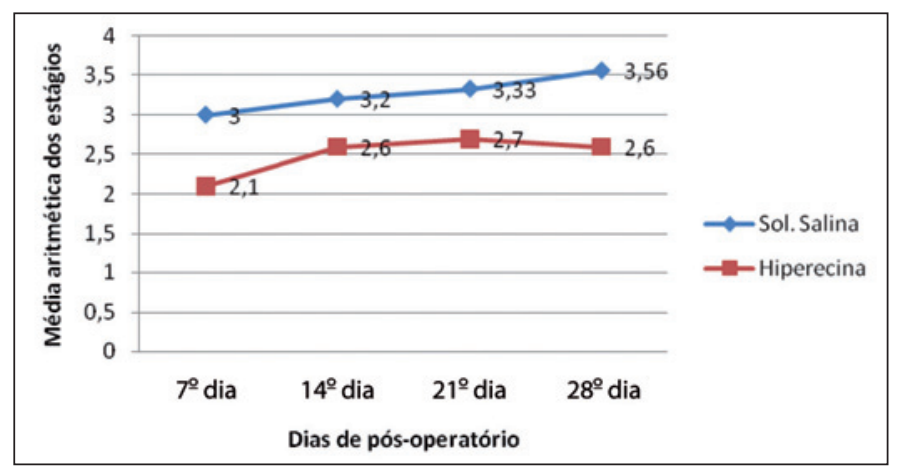

Figura 2 - Comparação de médias aritméticas

Em um modelo de retinopatia induzida por oxigênio em ratos, o tratamento com hiperecina via sonda gástrica reduziu o grau de neovascularização da retina ${ }^{(27)}$.

Utilizamos a hiperecina com dose de $10 \mu \mathrm{M}$, conforme sugerido por Tahara et al. ${ }^{(22)}$, que demonstraram resultados similares entre a dose de $10 \mu \mathrm{M}$ e $100 \mu \mathrm{M}$, com menor possibilidade de toxicidade.

Houve diferença estatisticamente significativa entre os dois grupos quando compararmos as posições da classificação de Hida et al. ${ }^{(21)}$ no $28^{\circ}$ dia de pós-operatório. Os autores sugerem que a partir do estágio 3 haveria um envolvimento extrarretiniano pelas membranas do PVR formado, sendo considerado a doença propriamente dita ${ }^{(21)}$.

Acreditamos que uma única dose da droga possa ter inibido os eventos iniciais da PVR, com isso atuando no "gatilho" do processo e sem efeitos em longo prazo sobre a homeostase dos tecidos retinianos.
A proteinoquinase $\mathrm{C}$ está presente nos vários compartimentos celulares, e em várias fases do metabolismo retiniano ${ }^{(28)}$; e sua inibição poderia acarretar efeitos adversos indesejáveis que devem ser avaliados em outro estudo.

\section{CONCLUSÕES}

O modelo experimental, utilizando a dispase (dose de 0,05 U diluída em $0,1 \mathrm{ml}$ ) associada à diatermia da retina (potência de $10 \%$ e 12 disparos) induz ao descolamento de retina e formação de membranas contráteis (PVR) em coelhos adultos pigmentados.

A utilização da hiperecina ( $10 \mu \mathrm{M}$ em $0,1 \mathrm{ml}$ via pars plana) em coelhos submetidos ao modelo experimental utilizando dispase e diatermia da retina reduziu, de forma estatisticamente significativa, a formação de PVR.

Mais estudos são necessários para melhor embasamento teórico do uso da hiperecina como tratamento de proliferação vitreorretiniana.

\section{ABSTRACT}

Purpose: To produce proliferative vitreoretinopathy (PVR) in an animal ocular trauma model. To evaluate the inhibition of (PVR) emergence and progression by hypericin. Methods: Experimental Study. Nineteen pigmented male adult rabbits weighing between 2,000 and 3,000 grams were used in this study. All of them were submitted to trauma model with dispase and retinal diathermy to induce PVR membranes formation. They were randomly assigned to receive hypericin $(10 \mu \mathrm{M}$ in $0.1 \mathrm{ml})$ or saline solution $(0.1 \mathrm{ml})$ as placebo. They 
were evaluated clinically in the seventh, fourteenth, twentyfirst and twenty-eighth postoperative days with indirect ophthalmoscopy and digital color retinography. The PVR degree was classified according to Hida (0 to 7). Results: Membranes formation was present in $79 \%$ of the eyes; being $100 \%$ in the eyes of placebo group and $60 \%$ in the eyes of treatment group (hypericin). The comparison between PVR phases averages within the groups showed a statistically significant difference between the two groups, with a $p$ value of 0.0321 for Wilcoxon test. Conclusions: The trauma model with dispase and retinal diathermy produces vitreoretinal membranes. Hypericin was considered effective in PVR emergence and progression decrease.

Keywords: Vitreoretinopathy, proliferative/etiology; Vitreoretinopathy, proliferative/prevention \& control; Antidepressive agents/therapeutic use; Retinal detachment; Eye injuries; Wound healing; Disease models, animal; Rabbits

\section{REFERÊNCIAS}

1. Ryan SJ. Traction retinal detachment. XLIX Edward Jackson Memorial Lecture. Am J Ophthalmol. 1993;115(1):1-20. Review.

2. Pastor JC. Proliferative vitreoretinopathy: an overview. Surv Ophthalmol. 1998;43(1):3-18.

3. Girard P, Mimoun G, Karpouzas I, Montefiore G. Clinical risk factors for proliferative vitreoretinopathy after retinal detachment surgery. Retina. 1994; 14(5):417-24.

4. Aaberg TM. Management of anterior and posterior proliferative vitreoretinopathy. XLV. Edward Jackson memorial lecture. Am J Ophthalmol. 1988;106(5): 519-32. Review.

5. Lewis H, Aaberg TM, Abrams GW, McDonalds HR, Williams GA, Mieler WF. Subretinal membranes in proliferative vitreoretinopathy. Ophthalmology. 1989;96(9):1403-14; discussion 1414-5.

6. Federman JL, Eagle RC Jr. Extensive peripheral retinectomy combined with posterior 360 degrees retinotomy for retinal reattachment in advanced proliferative vitreoretinopathy cases. Ophthalmology. 1990;97(10):1305-20.

7. Lewis H, Aaberg TM, Abrams GW. Causes of failure of the initial vitreoretinal surgery for severe proliferative vitreoretinopathy. Am J Ophthalmol. 1991; 111(1):8-14.

8. Lewis H, Aaberg TM. Causes of failure after repeat vitreoretinal surgery for recurrent proliferative vitreoretinopathy. Am J Ophthalmol. 1991;111(1):15-9.

9. Glaser BM, Carter JB, Kuppermann BD, Michels RG. Perfluoro-octane in the treatment of giant tears with proliferative vitreoretinopathy. Ophthalmology. 1991;98(11):1613-21.

10. Coll GE, Chang S, Sun J, Wieland MR, Berrocal MH. Perfluorcarbon liquid in the management of retinal detachment with proliferative vitreoretinopathy. Ophthalmology. 1995;102(4):630-8; discussion 638-9.
11. Stern WH, Lewis GP, Erickson PA, Guerin CJ, Anderson DH, Fisher SK, O'Donnell JJ. Fluorouracil therapy for proliferative vitreoretinopathy after vitrectomy. Am J Ophthalmol. 1983;96(1):33-42.

12. Wiedemann P, Hilgers RD, Bauer P, Heimann K. Adjunctive daunorubicin in the treatment of proliferative vitreoretinopathy: results of a multicenter clinical trial. Daunomycin Study Group. Am J Ophthalmol. 1998;126(4):550-9.

13. Kon CH, Occleston NL, Foss A, Sheridan C, Aylward GW, Khaw PT. Effects of single, short-term exposures of human retinal pigment epithelial cells to thiotepa or 5-fluorouracil: implications for the treatment of proliferative vitreoretinopathy. Br J Ophthalmol. 1998;82(5):554-60.

14. Yang CS, Khawly JA, Hainsworth DP, Chen SN, Ashton P, Guo H, Jafe GJ. An intravitreal sustained-release triamcinolone and 5-fluorouracil codrug in the treatment of experimental proliferative vitreoretinopathy. Arch Ophthalmol. 1998;116(1):69-77.

15. Ozerdem U, Mach-Hofacre B, Keefe K, Pham T, Soules K, Appelt K, Freeman WR. The effect of prinomastat (AG3340), a synthetic inhibitor of matrix metalloproteinases, on posttraumatic proliferative vitreoretinopathy. Ophthalmic Res. 2001;33(1):20-3.

16. Asaria RH, Kon CH, Bunce C, Charteris DG, Wong D, Khaw PT, Aylward GW. Adjuvant 5-fluorouracil and heparin prevents proliferative vitreoretinopathy: Results from a randomized, double-blind, controlled clinical trial. Ophthalmology. 2001; 108(7):1179-83. Comment in: Ophthalmology. 2001;108(7):1177-8. Ophthalmology. 2002;109(5):829-30; author reply 830.

17. Tano Y, Sugita G, Abrams G, Machemer R. Inhibition of intraocular proliferations with intravitreal corticosteroids. Am J Ophthalmol. 1980;89(1):131-6.

18. Hui YN, Hu D. Prevention of experimental proliferative vitreoretinopathy with daunomycin and triamcinolone based on the time course of the disease. Graefes Arch Clin Exp Ophthalmol. 1999;237(7):601-5.

19. Yoshida A, Elner SG, Bian ZM, Elner VM. Induction of interleukin-8 in human retinal pigment epithelial cells after denuding injury. Br J Ophthalmol. 2001; 85(7):872-6.

20. Blumenkranz MS, Ophir A, Claflin AJ, Hajek A. Fluorouracil for the treatment of massive periretinal proliferation. Am J Ophthalmol. 1982;94(4):458-67.

21. Hida T, Chandler DB, Sheta SM. Classification of the stages of proliferative vitreoretinopathy in a refined experimental model in the rabbit eye. Graefes Arch Clin Exp Ophthalmol. 1987;225(4):303-7.

22. Tahara YR, Sakamoto TR, Oshima YR, Ishibashi TR, Inomata HR, Murata TR, et al. The antidepressant hypericin inhibits progression of experimental proliferative vitreoretinopathy. Curr Eye Res. 1999;19(4):323-9.

23. Gao Q, Hui Y, Wang Y. [Effects of hypericin on traumatic proliferative vitreoretinopathy in rabbits]. Yan Ke Xue Bao. 2002;18(4):240-5. Chinese.

24. Alonso JR. Tratado de fitomedicina: bases clinicas y farmacológicas. Buenos Aires: Isis Ediciones S.R.L.; 1998

25. Agostinis P, Donella-Deana A, Cuveele J, Vandenbogaerde A, Sarno S, Merlevede $\mathrm{W}$, de Witte P. A comparative analysis of photosensitized inhibition of growth-factor regulated protein kinases by hypericin-derivatives. Biochem Biophys Res Commun. 1996;220(3):613-7.

26. Frenzel EM, Neely KA, Walsh AW, Cameron JD, Gregerson DS. A new model of proliferative vitreoretinopathy. Invest Ophthal Vis Sci. 1998;39(11):2157-64.

27. Higuchi A, Yamada H, Yamada E, Jo N, Matsumura M. Hypericin inhibits pathological retinal neovascularization in a mouse model of oxygen-induced retinopathy. Mol Vis. 2008;14:249-54.

28. Sheu SJ, Sakamoto T, Osusky R, Wang HM, Ogden TE, Ryan SJ, et al. Transforming growth factor-beta regulates human retinal pigment epithelial cell phagocytosis by influencing a protein kinase C-dependent pathway. Graefes Arch Clin Exp Ophthalmol. 1994;232(11):695-701. 